\title{
Automatic Parameter Prediction for Image Denoising Algorithms using Perceptual Quality Features
}

\author{
Anish Mittal, Anush K. Moorthy and Alan C. Bovik \\ The University of Texas at Austin Austin,Texas-78712,USA.
}

\begin{abstract}
A natural scene statistics (NSS) based blind image denoising approach is proposed, where denoising is performed without knowledge of the noise variance present in the image. We show how such a parameter estimation can be used to perform blind denoising by combining blind parameter estimation with a state-of-the-art denoising algorithm. ${ }^{1}$ Our experiments show that for all noise variances simulated on a varied image content, our approach is almost always statistically superior to the reference BM3D implementation in terms of perceived visual quality at the $95 \%$ confidence level.
\end{abstract}

\section{INTRODUCTION}

Image denoising has long been an interesting problem in the image processing community. This is natural owing to the way images are captured by the sensor and since noise is an integral part of the process. Hence, the problem has been well addressed in literature. ${ }^{1-21}$ The recent significant improvements achieved in denoising helped camera manufacturers to deploy mega pixel image sensors in small handheld devices and smart phones. This has also pushed the foot print of sensors to upper limits increasing the amount of sensor noise, consequently making problem more salient and interesting for image processing researchers.

Although recent denoising algorithms perform remarkably well, most of them require certain parameters to be set a priori, usually in an ad hoc fashion. Some empirical methods have been proposed in this direction that make use of L-curve methods, ${ }^{22-25}$ discrepancy principle ${ }^{26}$ and cross validation ${ }^{26-31}$ for parameter optimization. While these methods have led to better denoising performance, simply accounting for nature of visual content has led to better progress. Efforts have been directed towards optimizing the quality of estimated signals using the mean squared error. The pristine reference generally unavailable apriori, it must be replaced by other estimates not requiring the use of reference image, for example Stein's unbiased risk estimate. ${ }^{32-36}$ Given that the mean squared error is a poor measure of visual quality ${ }^{37}$ where performance is defined in terms of human judgements of image quality, a systematic perception based quality assessment approach would lead to improved performance. Towards this end, SSIM $^{38}$ based estimator was used for image denoising. ${ }^{39}$

Here we propose a natural scene statistics (NSS) based blind image denoising approach that seeks to reduce the amount of noise in a corrupted image without knowledge of the noise variance. We demonstrate how NSSbased parameter estimation may be used to create a blind denoising algorithm by combining blind parameter estimation with a state-of-the-art denoising algorithm. ${ }^{1}$ Even though the current work discusses the estimation of the noise variance parameter only, the approach can be used to estimate other parameters, depending on the unknown parameters of the image denoising approach it is used with.

We show that our blind parameter estimation procedure results in higher quality denoised images than the baseline for a wide range of noise variance values. The closest method in concept to our work is the one in, ${ }^{21}$ where the content of the image was used to predict noise variance. While the work $i^{21}$ is interesting, the approach is exhaustive and computationally intensive. $\mathrm{In},{ }^{21}$ the image is denoised multiple times using different values of the noise variance and the quality of each denoised image is estimated using their proposed no reference content-evaluation algorithm. The best image from this set is finally selected as the denoised image. Further, the limited evaluation presented in ${ }^{21}$ makes it difficult to judge the algorithm performance in a general-case scenario.

The paper is structured as follows: we first give details about the dataset used for our experiments and then explain a learning based blind parameter estimation approach that we use to create a blind image denoising approach. We then report experimental results and conclude with ideas about future work.

Further author information: (Send correspondence to Anish Mittal) : E-mail: mittal.anish@gmail.com 


\section{DATABASE}

A total of 300 reference images from the Berkley image segmentation database ${ }^{40}$ were randomly selected. Dataset has both portrait and landscape images of size $321 \times 481$ and $481 \times 321$ respectively.

Ten different levels of Gaussian noise were simulated using MATLAB's imnoise command resulting in 3000 distorted images. The distortion level represented by noise variance was uniformly sampled on a log-scale between 0.001 and 0.05. 1000 images were used for training phase and 2000 for testing phase. Train and test split was done to ensure that the content is disjoint among the two classes. Figure 1 shows sample images and their noisy versions with $\sigma=0.0316$ and $\sigma=0.2507$.

\section{APPROACH}

Our approach to blind parameter estimation is learning-based, where the input parameter is estimated using natural scene statistic based features proposed elsewhere for the purpose of blind image quality assessment ${ }^{41}$. During the training phase, our model resembles the approach in. ${ }^{21}$ The algorithm that we use to denoise the image $^{1}$ requires that the user provide the noise variance as an input parameter. However, we have observed that the denoised image produced when the algorithm is provided with the correct noise variance often has lower perceived quality than one is produced using a different (although incorrect) noise variance. For example, in Fig. 2, we plot a noisy image and two denoised versions of it, using the correct noise variance to generate as the result in ${ }^{1}$ and the denoised result from our approach which predicts a different input noise variance parameter. Notice that the latter has better quality, as supported by the improved multi-scale structural similarity index $(\text { MS-SSIM) })^{42}$ scores.

In order to account for this discrepancy and to ensure that the denoised image has the highest visual quality, the training stage proceeds as follows. Given a large set of noisy images, we denoise each image with various values of the input (noise variance) parameter using, ${ }^{1}$ and evaluate its visual quality using MS-SSIM. ${ }^{42}$ Amongst the denoised set, we pick the image having the highest perceptual quality as gauged by MS-SSIM, ${ }^{42}$ and use the corresponding input parameter as training input to the blind parameter estimation algorithm. Notice how this procedure, apart from producing images with high visual quality, also does not require knowledge of the actual noise variance in the training set. These estimated input parameters act as target values for our regressor which maps our features onto the input parameter using the training set.

The statistical features used to estimate the input parameter are the same as those that we have previously used in developing a blind image quality assessment algorithm named Blind/Referenceless Image Spatial QUality Evaluator (BRISQUE) ${ }^{41}$ The features are based on pointwise statistics of locally normalized luminance signals and distribution of pairwise products of neighbouring locally normalized luminance signals. An AGGD (Asymmetric Generalized Gaussian Model) distribution is utilized to fit the coefficients of both point wise and pairwise product distributions. Parameters of the distribution are used as features. The approach computes features at two scales.

During the training phase, we learn a regression from these statistical features to the input parameter as described above, and use the trained regressor in the testing phase to predict the input parameter given an unseen noisy image. This is followed by denoising using the algorithm in ${ }^{1}$ (BM3D).

\section{EXPERIMENTS AND RESULTS}

We used an online available MATLAB implemention of BM3D algorithm. ${ }^{43}$ To map statistical features to noise variance, any good regressor can be used. In our current implementation, we used a support vector machine regressor (SVR). ${ }^{44}$ This has previously been shown to perform well for quality assessment. ${ }^{41}$ In our implementation, we used the LIBSVM package ${ }^{45}$ and the radial basis function (RBF) kernel.

We compare the quality of the resulting denoised image against the one obtained using the default implementation of the BM3D algorithm where quality of denoising is measured using MS-SSIM. In Fig. 3, we plot the mean quality and the associated errors at each noise level across the 2000 test images for our approach, as well as for the reference implementation of BM3D. 


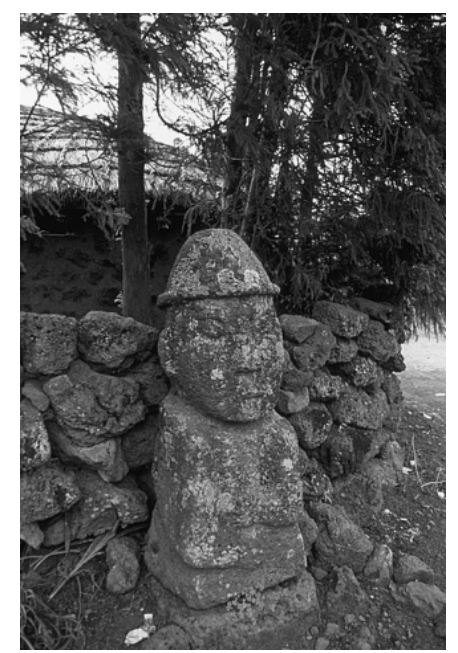

(a)

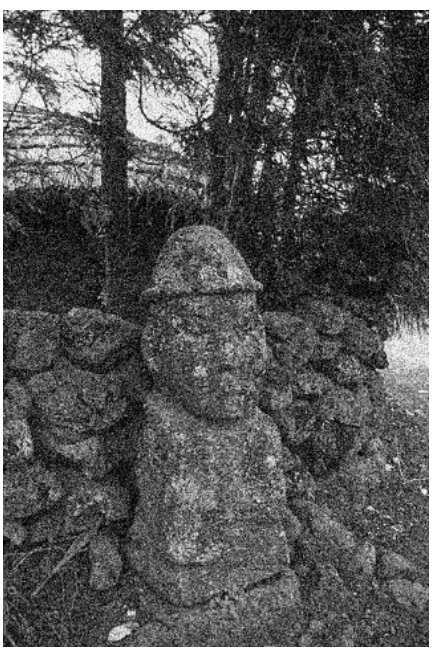

(c)

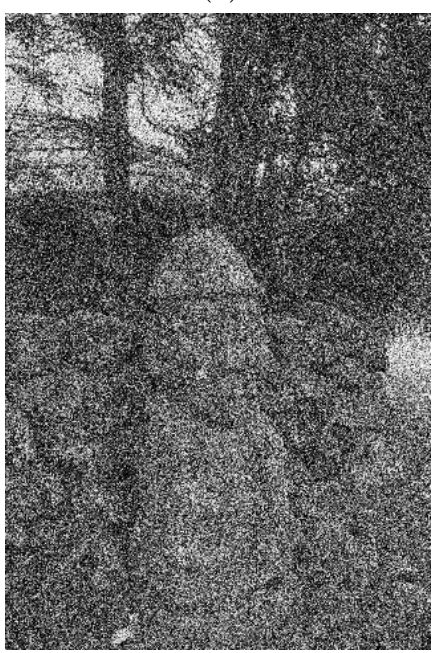

(e)

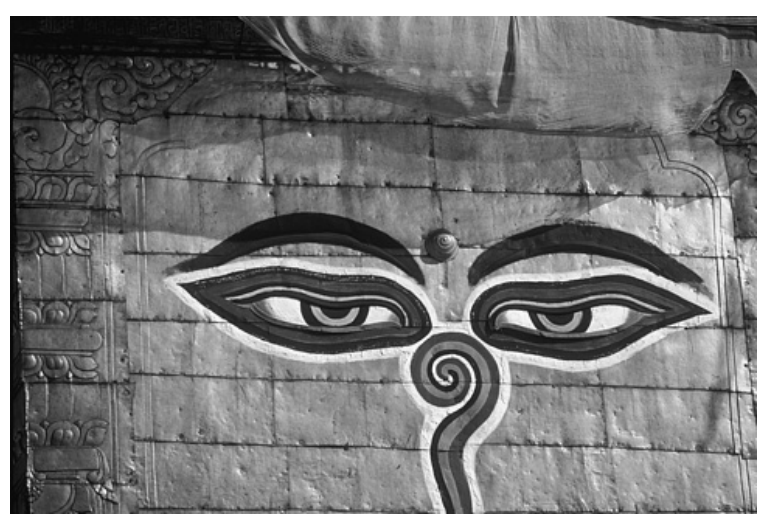

(b)

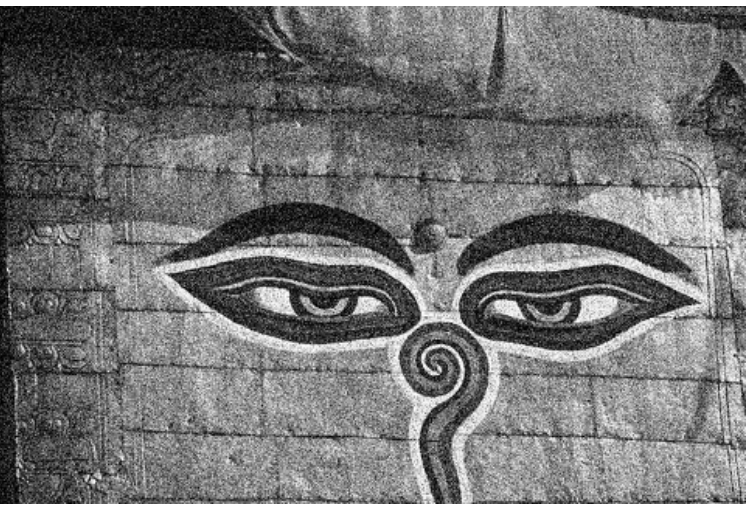

(d)

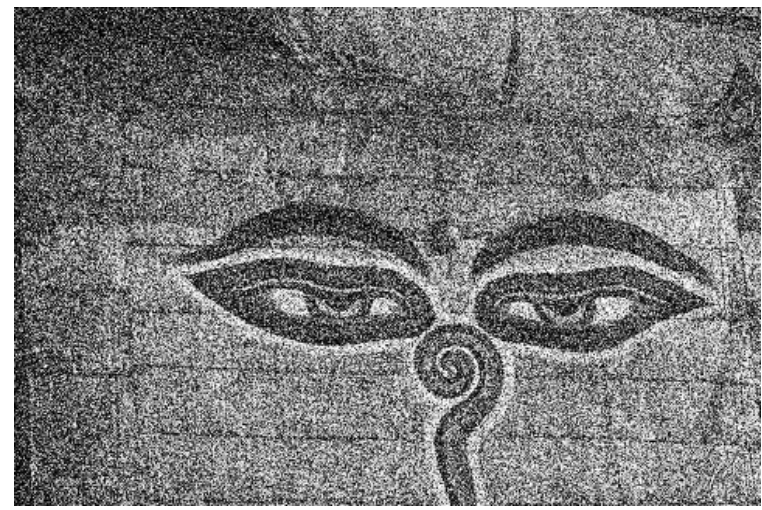

(f)

Figure 1. (a) and (b) show sample portrait and landscape images, (c)and (d) shows noisy versions of them with $\sigma=0.0316$ and (e)and (f) with $\sigma=0.2507$ respectively 


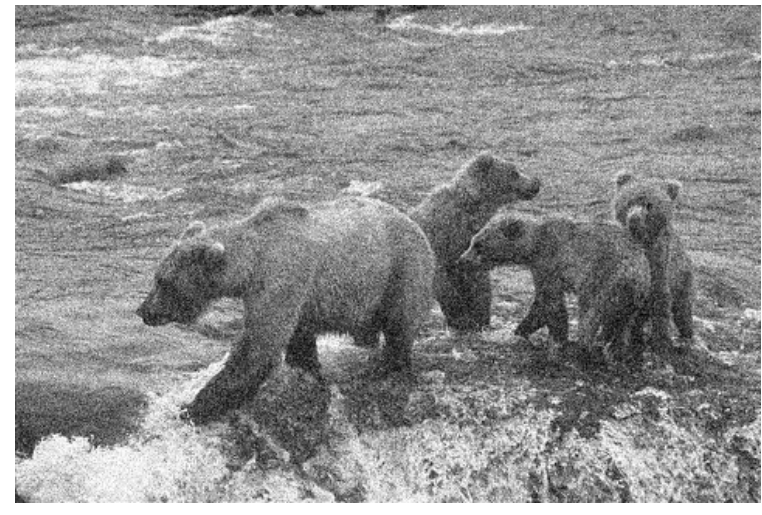

(a)

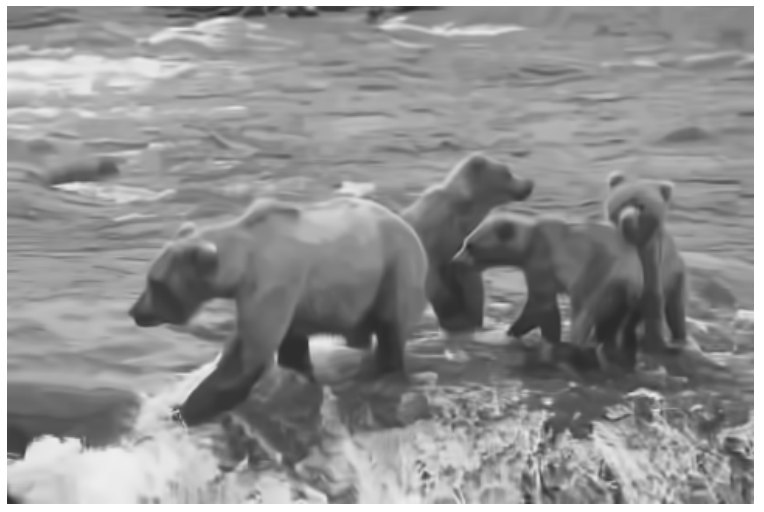

(b)

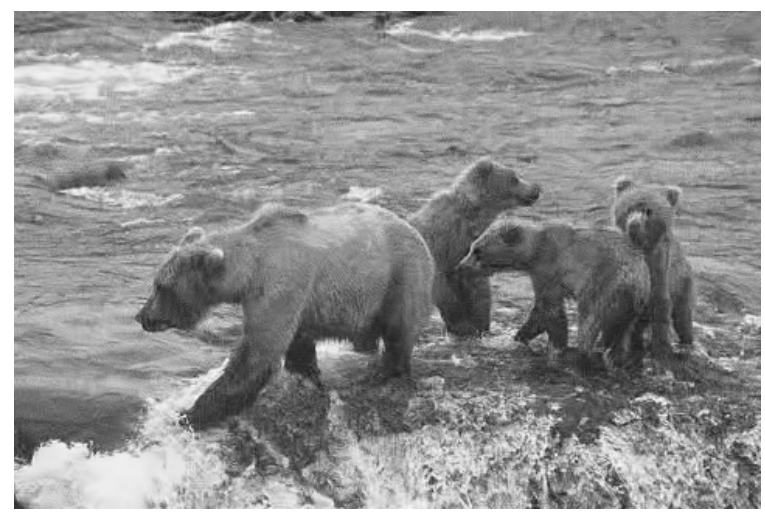

(c)

Figure 2. Accurate noise variance as input to the algorithm in ${ }^{21}$ produces poorer quality denoised images: (a) Noisy Image $(\sigma=0.0158$, MS-SSIM $=0.9063)$, (b) Denoised with $\sigma=0.0158($ MS-SSIM $=0.9176)$ and (c) Denoised with $\sigma=0.0040$ $(\mathrm{MS}-\mathrm{SSIM}=0.9480)$

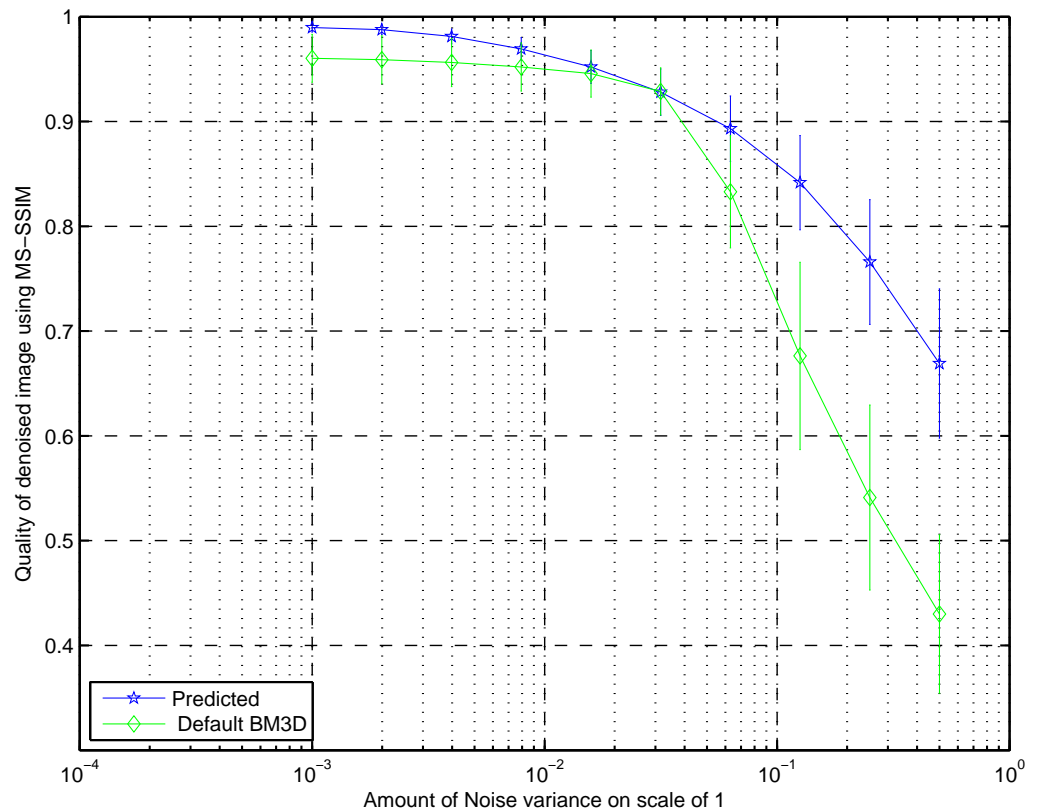

Figure 3. Figure shows the mean quality and associated errors at each noise level across 2000 test images for our approach as well as the reference implementation of BM3D 
We also analyzed whether the differences observed in the quality of the denoised images between our approach and the reference BM3D implementation are statistically significant using the t-test. ${ }^{46}$ Our analysis indicates that for all noise variances simulated in this study, our approach is statistically superior to the reference BM3D implementation in terms of perceived visual quality at the $95 \%$ confidence level, excepting when the noise variance is at 0.0316 - where the two approaches are statistically indistinguishable.

\section{CONCLUSION AND FUTURE WORK}

A natural scene statistics (NSS) based blind image denoising approach was proposed by combining blind parameter estimation with a state-of-the-art denoising algorithm. ${ }^{1}$ It was shown to perform statistically superior to the reference BM3D implementation, where performance was defined in terms of perceived visual quality at the $95 \%$ confidence level, excepting when the noise variance is at 0.0316 - where the two approaches are statistically indistinguishable.

Current work has addressed estimation of noise variance parameter but our approach is generic enough to be used for any parameter estimation depending on the input required by image denoising approach it is used with. Future work would involve parameter estimation for other denoising algorithms. Also, we have only used images afflicted with gaussian noise for our present analysis. It would be of interest to discover how well BRISQUE features ${ }^{41}$ would perform when estimating parameters representative of other distortions, for instance JPEG quantization distortion, and subsequently using it for blind image deblocking.

\section{REFERENCES}

[1] Dabov, K., Foi, A., Katkovnik, V., and Egiazarian, K., "Image denoising by sparse 3-d transform-domain collaborative filtering," Transactions on Image Processing 16(8), 2080-2095 (2007).

[2] Portilla, J., Strela, V., Wainwright, M., and Simoncelli, E. P., "Image denoising using scale mixtures of gaussians in the wavelet domain," Transactions on Image Processing 12(11), 1338-1351 (2003).

[3] Rabbani, H., Vafadoost, M., Selesnick, I., and Gazor, S., "Image denoising based on a mixture of bivariate gaussian models in complex wavelet domain," in [International Summer School on Medical Devices and Biosensors], 149-153, IEEE (2006).

[4] Rabbani, H., Vafadust, M., Selesnick, I., and Gazor, S., "Image denoising employing a mixture of circular symmetric laplacian models with local parameters in complex wavelet domain," in [International Conference on Acoustics, Speech and Signal Processing], 1, 805-808, IEEE (2007).

[5] Le Pennec, E. and Mallat, S., "Sparse geometric image representations with bandelets," Transactions on Image Processing 14(4), 423-438 (2005).

[6] Starck, J. L., Candes, E. J., and Donoho, D. L., "The curvelet transform for image denoising," Transactions on Image Processing 11(6), 670-684 (2002).

[7] Foi, A., Katkovnik, V., and Egiazarian, K., "Pointwise shape-adaptive dct for high-quality denoising and deblocking of grayscale and color images," Transactions on Image Processing 16(5), 1395-1411 (2007).

[8] Buades, A., Coll, B., and Morel, J. M., "A review of image denoising algorithms, with a new one," Multiscale Modeling and Simulation 4(2), 490-530 (2006).

[9] Brox, T., Kleinschmidt, O., and Cremers, D., "Efficient nonlocal means for denoising of textural patterns," Transactions on Image Processing 17(7), 1083-1092 (2008).

[10] Dauwe, A., Goossens, B., Luong, H. Q., and Philips, W., "A fast non-local image denoising algorithm," in [Proceedings of SPIE], 6812, 681210, IEEE (2008).

[11] Mahmoudi, M. and Sapiro, G., "Fast image and video denoising via nonlocal means of similar neighborhoods," Signal Processing Letters 12(12), 839-842 (2005).

[12] Orchard, J., Ebrahimi, M., and Wong, A., "Efficient nonlocal-means denoising using the svd," in [International Conference on Image Processing], 1732-1735, IEEE.

[13] Wang, J., Guo, Y., Ying, Y., Liu, Y., and Peng, Q., "Fast non-local algorithm for image denoising," in [International Conference on Image Processing], 1429-1432, IEEE (2006).

[14] Kervrann, C. and Boulanger, J., "Optimal spatial adaptation for patch-based image denoising," Transactions on Image Processing 15(10), 2866-2878 (2006). 
[15] Takeda, H., Farsiu, S., and Milanfar, P., "Kernel regression for image processing and reconstruction," Transactions on Image Processing 16, 349-366 (2007).

[16] Elad, M. and Aharon, M., "Image denoising via sparse and redundant representations over learned dictionaries," Transactions on Image Processing 15(12), 3736-3745 (2006).

[17] Chatterjee, P. and Milanfar, P., "Clustering-based denoising with locally learned dictionaries," Transactions on Image Processing 18(7), 1438-1451 (2009).

[18] Chatterjee, P. and Milanfar, P., "Is denoising dead," Transactions on Image Processing 19(4), 895-911 (2010).

[19] Van De Ville, D. and Kocher, M., "Sure-based non-local means," Signal Processing Letters 16(11), 973-976 (2009).

[20] Salmon, J., "On two parameters for denoising with non-local means," Signal Processing Letters 17(3), 269-272 (2010).

[21] Zhu, X. and Milanfar, P., "Automatic parameter selection for denoising algorithms using a no-reference measure of image content," Transactions on Image Processing 19(12), 3116-3132 (2010).

[22] Hansen, P. C., "Analysis of discrete ill-posed problems by means of the l-curve," SIAM review 34(4), 561-580 (1992).

[23] Hansen, P. C. and Oĺeary, D. P., "The use of the l-curve in the regularization of discrete hx-posed problems," SIAM Journal on Scientific Computing .

[24] Regińska, T., "A regularization parameter in discrete ill-posed problems," SIAM Journal on Scientific Computing 17(3), 740-749 (1996).

[25] Oraintara, S., Karl, W., Castanon, D., and Nguyen, T., "A method for choosing the regularization parameter in generalized tikhonov regularized linear inverse problems," in [International Conference on Image Processing], 1, 93-96, IEEE (2000).

[26] Karl, W. C., "Regularization in image restoration and reconstruction," Handbook of Image and Video Processing , 141-160 (2000).

[27] Craven, P. and Wahba, G., "Smoothing noisy data with spline functions," Numerische Mathematik 31(4), 377-403 (1978).

[28] Golub, G. H., Heath, M., and Wahba, G., "Generalized cross-validation as a method for choosing a good ridge parameter," Technometrics , 215-223 (1979).

[29] Nychka, D., "Bayesian confidence intervals for smoothing splines," Journal of the American Statistical Association, 1134-1143 (1988).

[30] Thompson, A. M., Brown, J. C., Kay, J. W., and Titterington, D. M., "A study of methods of choosing the smoothing parameter in image restoration by regularization," Transactions on Pattern Analysis and Machine Intelligence 13(4), 326-339 (1991).

[31] Galatsanos, N. P. and Katsaggelos, A. K., "Methods for choosing the regularization parameter and estimating the noise variance in image restoration and their relation," Transactions on Image Processing $\mathbf{1}(3)$, 322-336 (1992).

[32] Ramani, S., Blu, T., and Unser, M., "Monte-carlo sure: A black-box optimization of regularization parameters for general denoising algorithms," Transactions on Image Processing 17(9), 1540-1554 (2008).

[33] Blu, T. and Luisier, F., "The sure-let approach to image denoising," Transactions on Image Processing 16(11), 2778-2786 (2007).

[34] Luisier, F., Blu, T., and Unser, M., "A new sure approach to image denoising: Interscale orthonormal wavelet thresholding," Transactions on Image Processing 16(3), 593-606 (2007).

[35] Zhang, X. and Desai, M., "Adaptive denoising based on sure risk," Signal Processing Letters 5(10), 265-267 (1998).

[36] Donoho, D. and Johnstone, I., "Adapting to unknown smoothness via wavelet shrinkage," Journal of the american statistical association, 1200-1224 (1995).

[37] Wang, Z. and Bovik, A. C., "Mean squared error: love it or leave it? A new look at signal fidelity measures," Signal Processing Magazine 26(1), 98-117 (2009).

[38] Wang, Z., Bovik, A. C., Sheikh, H. R., and Simoncelli, E. P., "Image quality assessment: From error visibility to structural similarity," Transactions on Image Processing 13(4), 600-612 (2004). 
[39] Channappayya, S. S., Bovik, A. C., and Heath, R. W., "A linear estimator optimized for the structural similarity index and its application to image denoising," in [International Conference on Image Processing], 2637-2640, IEEE (2006).

[40] Martin, D., Fowlkes, C., Tal, D., and Malik, J., "A database of human segmented natural images and its application to evaluating segmentation algorithms and measuring ecological statistics," in [International Conference on Computer Vision], 2, 416-423 (2001).

[41] Mittal, A., Moorthy, A. K., and Bovik, A. C., "Blind/referenceless image spatial quality evaluator," Asilomar Conference on Signals, Systems and Computers (2011).

[42] Wang, Z., Simoncelli, E. P., and Bovik, A. C., "Multiscale structural similarity for image quality assessment," in [Asilomar Conference on Signals, Systems and Computers], 2, 1398-1402, IEEE (2003).

[43] Dabov, K., Foi, A., Katkovnik, V., and Egiazarian, K., "BM3D Code [Online]Available:." http://www.cs.tut.fi/ foi/GCF-BM3D/.

[44] Schölkopf, B., Smola, A. J., Williamson, R. C., and Bartlett, P. L., "New support vector algorithms," Neural computation 12(5), 1207-1245 (2000).

[45] Chang, C. C. and Lin, C. J., "LIBSVM: a library for support vector machines," (2001).

[46] Hedges, L. V. and Olkin, I., [Statistical methods for meta-analysis], Academic Press New York: (1985). 